\title{
A NOTE ON CONTRACTIVE MAPPINGS
}

E. RAKOTCH ${ }^{1}$

A well-known theorem of Banach [1] states: if $A$ is a mapping of a complete metric space $X$ into itself, and there exists a number $0<\alpha<1$ such that for every two points $x, y \in X$

$$
\rho(A x, A y) \leqq \alpha \rho(x, y),
$$

then there exists a unique fixed point of $A$, i.e. a point $x$ such that $A x=x$.

The problem of defining a family of functions $F=\{\alpha(x, y)\}$ satisfying $0 \leqq \alpha(x, y)<1, \sup \alpha(x, y)=1$ and such that Banach's theorem holds when the constant $\alpha$ is replaced with any $\alpha(x, y) \in F$, was suggested to the author by Professor H. Hanani, and two theorems to that effect are proved in the present paper. In the first, additional conditions requiring compactness of the mapping are imposed; the second holds for any complete metric space, irrespective of the compactness of the mapping.

Definition 1. A mapping $A$ of a metric space $X$ into itself is said to be contractive if for every two distinct points $x, y \in X$

$$
\rho(A x, A y)<\rho(x, y) .
$$

A contractive mapping is clearly continuous, and if such a mapping has a fixed point, then this fixed point is obviously unique.

Definition 2. Denote by $F_{1}$ the family of functions $\alpha(x, y)$ satisfying the following conditions:

(1) $\alpha(x, y)=\alpha(\rho(x, y))$, i.e., $\alpha$ is dependent on the distance between $x$ and $y$ only.

(2) $0 \leqq \alpha(\rho)<1$ for every $\rho>0$.

(3) $\alpha(\rho)$ is a monotonically decreasing function of $\rho$.

To prove the first theorem, use is made of the following theorem of Edelstein [2]:

Theorem (M. Edelstein). Let $X$ be a metric space and $A$ a contractive mapping of $X$ into itself such that there exists a point $x \in X$ whose sequence of iterates $\left\{A^{n} x\right\}$ contains a convergent subsequence $\left\{A^{n_{i}} x\right\}$; then $\xi=\lim _{i \rightarrow \infty} A^{n_{i}} x \in X$ is a unique fixed point.

Theorem 1. If $A$ is a contractive mapping of a metric space $X$ into

Received by the editors March 22, 1961.

1 This note is part of the author's thesis for the degree of Master of Science, submitted to the Department of Mathematics of the Technion, I.I.T., Haifa. 
itself, and there exist a subset $M \subset X$ and a point $x_{0} \in M$ such that

$$
\rho\left(x, x_{0}\right)-\rho\left(A x, A x_{0}\right) \geqq 2 \rho\left(x_{0}, A x_{0}\right) \quad \text { for every } x \in X \backslash M
$$

and $A$ maps $M$ into a compact subset of $X$, then there exists a unique fixed point.

Proof. Suppose $A x_{0} \neq x_{0}$ and let

$$
x_{n}=A^{n} x_{0}, \quad n=1,2, \cdots,
$$

i.e.,

$$
x_{n+1}=A x_{n},
$$$$
n=0,1, \cdots .
$$

$A$ maps $M$ into a compact set by assumption; thus, to obtain the theorem as a direct consequence of Edelstein's theorem, it is sufficient to show that $x_{n} \in M$ for every $n$.

Since $A$ is a contractive mapping, the sequence $\rho\left(x_{n}, x_{n+1}\right)$ is, by (2), nonincreasing, and by $A x_{0} \neq x_{0}$ it follows that

$$
\rho\left(x_{n}, x_{n+1}\right)<\rho\left(x_{0}, x_{1}\right), \quad n=1,2, \cdots .
$$

By the triangle inequality

$$
\rho\left(x_{0}, x_{n}\right) \leqq \rho\left(x_{0}, x_{1}\right)+\rho\left(x_{1}, x_{n+1}\right)+\rho\left(x_{n}, x_{n+1}\right) .
$$

Thus by (2) and (3)

$$
\rho\left(x_{0}, x_{n}\right)-\rho\left(A x_{0}, A x_{n}\right)<2 \rho\left(x_{0}, A x_{0}\right),
$$

and by (1) it follows that $x_{n} \in M$ for every $n$; hence the theorem follows.

Corollary. If $A$ is a contractive mapping such that there exists a point $x_{0} \in X$ satisfying

$$
\rho\left(A x, A x_{0}\right) \leqq \alpha\left(x, x_{0}\right) \rho\left(x, x_{0}\right)
$$

for every $x \in X$, where $\alpha(x, y)=\alpha(\rho(x, y)) \in F_{1}$, and $A \operatorname{maps}^{2} S\left(x_{0}, r\right)$ with

$$
r=\frac{2 \rho\left(x_{0}, A x_{0}\right)}{1-\alpha\left(2 \rho\left(x_{0}, A x_{0}\right)\right)}
$$

into a compact subset of $X$, then there exists a unique fixed point of $A$.

Indeed, take in Theorem $1, M=S\left(x_{0}, r\right)$; then by (II), the monotonicity of $\alpha(\rho)$ and $r \geqq 2 \rho\left(x_{0}, A x_{0}\right)$, it follows that if $\rho\left(x, x_{0}\right) \geqq r$, then

$$
{ }^{2} S\left(x_{0}, r\right)=\left\{x \mid \rho\left(x, x_{0}\right)<r\right\} \text {. }
$$




$$
\begin{aligned}
\rho\left(x, x_{0}\right)-\rho\left(A x, A x_{0}\right) & \geqq \rho\left(x, x_{0}\right)-\alpha\left(\rho\left(x, x_{0}\right)\right) \rho\left(x, x_{0}\right) \\
& =\left[1-\alpha\left(\rho\left(x, x_{0}\right)\right)\right] \rho\left(x, x_{0}\right) \geqq[1-\alpha(r)] r \\
& \geqq\left[1-\alpha\left(2 \rho\left(x_{0}, A x_{0}\right)\right)\right] r=2 \rho\left(x_{0}, A x_{0}\right),
\end{aligned}
$$

i.e., (1) holds.

REMARK 1 . If $A$ is a completely continuous (not necessarily contractive) mapping, $X$ is a Banach space and $M \subset X$ is a bounded subset, then the existence of a fixed point follows from (1) by Schauder's fixed-point theorem [4], which states that every continuous mapping of a closed convex subset of a Banach space into a compact subset of itself has a fixed point.

Proof. $A$ being completely continuous, it is sufficient to show that $A$ maps some closed sphere into itself.

Denote

$$
\left\|A x_{0}-x_{0}\right\|=a
$$

by the triangle inequality

$$
\begin{aligned}
\left\|A x-x_{0}\right\| & \leqq\left\|A x-A x_{0}\right\|+\left\|A x_{0}-x_{0}\right\| \\
& =\left\|x-x_{0}\right\|-\left(\left\|x-x_{0}\right\|-\left\|A x-A x_{0}\right\|\right)+a .
\end{aligned}
$$

By (1) there exists a number $r_{0}$ such that $S\left(x_{0}, r_{0}\right) \supset M$ and $\left\|x-x_{0}\right\|$ $>r_{0}$ implies $\left\|x-x_{0}\right\|-\left\|A x-A x_{0}\right\| \geqq a$; hence for every $x$ such that $\left\|x-x_{0}\right\|>r_{0}$ we have

$$
\left\|A x-x_{0}\right\| \leqq\left\|x-x_{0}\right\| .
$$

$A$ being compact, there exists $R$ such that from $\left\|x-x_{0}\right\| \leqq r_{0}$ it follows $\left\|A x-x_{0}\right\| \leqq R$. Consequently $A$ maps the closed sphere $\tilde{S}\left(x_{0}, r\right):\left\|x-x_{0}\right\| \leqq r$, where $r=\max \left(r_{0}, R\right)$, into itself, which proves our proposition.

REMARK 2. The assumption

$$
\rho(A x, A y)<\rho(x, y)
$$

and compactness of $A$ are not sufficient for the existence of a fixed point, as it can be seen from the following example:

Let $X$ be the space of all real numbers, and define the function

$$
f(x)=\ln \left(1+e^{x}\right)
$$

Differentiating we obtain

$$
f^{\prime}(x)=\frac{e^{x}}{1+e^{x}}<1
$$

i.e., $f$ is a contractive mapping, and it is easy to see that $f$ has no fixed 
point although it is a compact mapping (even completely continuous).

THEOREM 2. Let $A$ be a contractive mapping of a complete metric space $X$ into itself such that there exists a subset $M \subset X$ and $a$ point $x_{0} \in M$ satisfying the following:

$$
\begin{array}{ll}
\rho\left(x, x_{0}\right)-\rho\left(A x, A x_{0}\right) \geqq 2 \rho\left(x_{0}, A x_{0}\right) & \text { for every } x \in X \backslash M, \\
\rho(A x, A y) \leqq \alpha(x, y) \rho(x, y) & \text { for every } x, y \in M,
\end{array}
$$

where

$$
\alpha(x, y)=\alpha(\rho(x, y)) \in F_{1} .
$$

Then there exists a unique fixed point.

Proof. Assume that $A x_{0} \neq x_{0}$ and define as before the sequence

$$
x_{n}=A^{n} x_{0}, \quad n=1,2, \cdots \text {. }
$$

By the same method as in Theorem 1 we obtain, using (4), that

$$
\rho\left(x_{n}, x_{n+1}\right)<\rho\left(x_{0}, x_{1}\right), \quad n=1,2, \cdots,
$$

and $x_{n} \in M$ for every $n$.

Now we prove that the sequence $\left\{x_{n}\right\}$ is bounded.

By (5) and the definition of $\left\{x_{n}\right\}$,

$$
\rho\left(x_{1}, x_{n+1}\right)=\rho\left(A x_{0}, A x_{n}\right) \leqq \alpha\left(\rho\left(x_{0}, x_{n}\right)\right) \rho\left(x_{0}, x_{n}\right)
$$

and by the triangle inequality,

$$
\rho\left(x_{0}, x_{n}\right) \leqq \rho\left(x_{0}, x_{1}\right)+\rho\left(x_{1}, x_{n+1}\right)+\rho\left(x_{n}, x_{n+1}\right) .
$$

Hence by (6) and (7)

$$
\left[1-\alpha\left(\rho\left(x_{0}, x_{n}\right)\right)\right] \rho\left(x_{0}, x_{n}\right)<2 \rho\left(x_{0}, x_{1}\right) .
$$

Now if $\rho\left(x_{0}, x_{n}\right) \geqq \rho_{0}$ for a given $\rho_{0}>0$, then by the monotonicity of $\alpha(\rho)$ it follows that $\alpha\left(\rho\left(x_{0}, x_{n}\right)\right) \leqq \alpha\left(\rho_{0}\right)$ and therefore

$$
\rho\left(x_{0}, x_{n}\right)<\frac{2 \rho\left(x_{0}, x_{1}\right)}{1-\alpha\left(\rho\left(x_{0}, x_{n}\right)\right)} \leqq \frac{2 \rho\left(x_{0}, x_{1}\right)}{1-\alpha\left(\rho_{0}\right)}=C \text {. }
$$

Hence

$$
\rho\left(x_{0}, x_{n}\right) \leqq R, \quad n=1,2, \cdots,
$$

where $R=\max \left(\rho_{0}, C\right)$, i.e., the sequence $\left\{x_{n}\right\}$ is bounded.

Now let $p>0$ be an arbitrary integer; then by (5)

$$
\rho\left(x_{k+1}, x_{k+p+1}\right) \leqq \alpha\left(x_{k}, x_{k+p}\right) \rho\left(x_{k}, x_{k+p}\right) .
$$

Hence, taking the product from $k=0$ to $k=n-1$ and dividing both sides by the same terms, we obtain 


$$
\rho\left(x_{n}, x_{n+p}\right) \leqq \rho\left(x_{0}, x_{p}\right) \prod_{k=0}^{n-1} \alpha\left(x_{k}, x_{k+p}\right)
$$

(the division by the same terms is correct, since, as is easily seen, the inequality holds also in the case where $\rho\left(x_{k+1}, x_{k+p+1}\right)=0$ for some $k<n-1)$ and by (8) it follows that

$$
\rho\left(x_{n}, x_{n+p}\right) \leqq R \prod_{k=0}^{n-1} \alpha\left(x_{k}, x_{k+p}\right) .
$$

To prove that $\left\{x_{n}\right\}$ is a Cauchy sequence, it is sufficient to show that for every $\epsilon>0$ there exists a number $N$, dependent on $\epsilon$ only (not on $p$ ) such that for every $p>0$ there is $\rho\left(x_{N}, x_{N+p}\right)<\epsilon$ (since the sequence $\rho\left(x_{n}, x_{n+p}\right)$ is nonincreasing).

If $\rho\left(x_{k}, x_{k+p}\right) \geqq \epsilon$ for $k=0,1, \cdots, n-1$, then by (5) (the monotonicity of $\alpha(\rho))$ we have $\alpha\left(x_{k}, x_{k+p}\right)=\alpha\left(\rho\left(x_{k}, x_{k+p}\right)\right) \leq \alpha(\epsilon)$, and by (9) it follows that

$$
\rho\left(x_{n}, x_{n+p}\right) \leqq R[\alpha(\epsilon)]^{n} .
$$

But $\alpha(\epsilon)<1$ and $[\alpha(\epsilon)]^{n} \rightarrow 0$ as $n \rightarrow \infty$, so there exists an integer $N$, independent of $p$, such that $\rho\left(x_{N}, x_{N+p}\right)<\epsilon$ for every $p>0$, which proves that $\left\{x_{n}\right\}$ is a Cauchy sequence.

By the completeness of $X$ it follows that there exists $x=\lim _{n \rightarrow \infty} x_{n}$ and by the continuity of $A, x$ is a fixed point.

Putting $M=X$, we obtain the

Corollary. If $\rho(A x, A y) \leqq \alpha(x, y) \rho(x, y)$ for every $x, y \in X$ (X complete), where $\alpha(x, y) \in F_{1}$, then there exists a unique fixed point.

REMark 3. If in Theorem 1 and Theorem $2 x_{0} \notin M$, then the existence of a fixed point follows at once by substituting $x=x_{0}$ in (1) and (4).

REMARK 4. The above corollary and Theorem 2 are generalizations of Banach's fixed-point theorem.

We now introduce the following definitions:

Definition 3. An operator $T$ defined on a domain $D$ of a normed space $X$ is said to satisfy Lipschitz conditions on this domain if there exists a constant $C$ such that

$$
\left\|T x_{1}-T x_{2}\right\| \leqq C\left\|x_{1}-x_{2}\right\|
$$

for all $x_{1}, x_{2} \in D$.

The infimum of all the constants $C$ satisfying (10), or

$$
\sup _{x_{1}, x_{2} \in D} \frac{\left\|T x_{1}-T x_{2}\right\|}{\left\|x_{1}-x_{2}\right\|}
$$


is ealled the Lipschitz norm of $T$ on $D$ and denoted by $\|T\|$.

Definition 4. An operator $T$ is said to be strictly contractive on a domain $D$ if $\||T|\|<1$.

With these definitions, E. H. Zarantonello [5] proved the following lemma in a Hilbert space:

LEMMA $1^{3}$ (CONTRACTION PRINCIPLE). If $T$ is strictly contractive on a sphere $S_{a}(y):\|x-y\| \leqq a$ about the point $y$, and if

$$
\|T y\| \leqq a(1-\|T\|)
$$

then the equation

$$
x=y+T x
$$

has a unique solution in $S_{a}(y)$ and

$$
\|x-y\| \leqq \frac{\|T y\|}{1-\|T\|} .
$$

This lemma is essentially a localization of Banach's fixed-point theorem.

By methods similar to those of Zarantonello the following theorems are arrived at:

Theorem 3 (LOCAlization of TheOREM 1). Let $T$ be contractive on a sphere $S\left(x_{0}, r\right): \rho\left(x, x_{0}\right) \leqq r$ in a linear metric space $X$ so that for every $x \in S\left(x_{0}, r\right)$ there is

$$
\rho\left(T x, T x_{0}\right) \leqq \alpha\left(x, x_{0}\right) \rho\left(x, x_{0}\right),
$$

where $\alpha(x, y) \in F_{1}$.

If

$$
\rho\left(T x_{0}, \theta\right) \leqq r \sup _{\rho \leqq r} \rho \alpha(\rho),
$$

and $T$ maps $S\left(x_{0}, r\right)$ into a compact subset of $X$, then the equation

$$
x=x_{0}+T x
$$

has exactly one solution in $S\left(x_{0}, r\right)$.

Theorem 4 (LOCAlization of TheOREM 2). Let $T$ be contractive on a sphere $S\left(x_{0}, r\right): \rho\left(x, x_{0}\right) \leqq r$ in a complete metric linear space so that for every $x, y \in S\left(x_{0}, r\right)$ there is

$$
\rho(T x, T y) \leqq \alpha(x, y) \rho(x, y),
$$

${ }^{8}$ A weaker formulation of this lemma, obtained by replacing the $\leqq$ sign in (11) by $<$, is a direct consequence of Theorem 1 of [3]. 
where $\alpha(x, y) \in F_{1}$.

If

$$
\rho\left(T x_{0}, \theta\right) \leqq r-\sup _{\rho \leqq r} \rho \alpha(\rho)
$$

then the equation

$$
x=x_{0}+T x
$$

has exactly one solution in $S\left(x_{0}, r\right)$.

The author wishes to thank Professor H. Hanani and Dr. M. Reichbach for guidance and help in preparing this paper.

\section{REFERENCES}

1. S. Banach, Sur les opérations dans les ensembles abstraits et leur applications aux equations intégrales, Fund. Math. 3 (1922), 160.

2. M. Edelstein, On fixed and periodic points under contraction mappings, J. London Math. Soc. (to appear).

3. T. H. Hildebrandt and L. M. Graves, Implicit functions and their differentials in general analysis, Trans. Amer. Math. Soc. 29 (1927), 127-153. 175.

4. J. Schauder, Der Fixpunktsatz in Funktionalräumen, Studia Math. 2 (1930),

5. E. H. Zarantonello, Solving functional equations by contractive averaging, Mathematical Research Center, Madison, Wisconsin, Technical Summary Report No. 160, June 1960.

Technion, Israel Institute of Technology, Haifa, Israel 